\title{
PERSPECTIVAS DE DESARROLLO DE LA ERGONOMÍA EN LA ARGENTINA: UNA MIRADA DESDE LA UNIVERSIDAD TECNOLÓGICA NACIONAL
}

\author{
PERSPECTIVES FOR ERGONOMICS DEVELOPMENT IN ARGENTINE: A VIEW FROM \\ THE NATIONAL TECHNOLOGICAL UNIVERSITY
}

\author{
Lucie Nouviale ${ }^{1}$ \\ Esther Doris Catalina Giraudo ${ }^{2}$
}

\begin{abstract}
Resumen: Este artículo aborda el desarrollo de la profesión en la Argentina a partir de un repaso histórico nacional de las publicaciones científicas, la legislación y la oferta de capacitaciones. Luego se analizó la totalidad de los 6o trabajos finales producidos entre los años 2014 y 2020 por alumnos formados en la carrera en ergonomía de la Universidad Tecnológica Nacional - Facultad Regional de Buenos Aires. El estudio se basó en la estadística descriptiva como método para identificar sus características más importantes y los resultados destacaron la industria manufacturera como principal rama de la actividad en la cual se desarrollaron. Las personas indagadas en el origen de estos trabajos fueron los tomadores de decisión (supervisores, jefes, gerentes y directores) y los expertos miembros de estas organizaciones (recursos humanos, medicina laboral, calidad, seguridad e higiene). Los objetivos de los trabajos realizados estuvieron orientados hacia la resolución de problemas existentes, la mayoría relacionados con el campo de la ergonomía física para disminuir los accidentes y los trastornos músculo esqueléticos en el trabajo. El artículo termina abriendo la reflexión sobre las estrategias para mejorar la oferta educativa y la calidad de la carrera en ergonomía a fin de acompañar el desarrollo de la profesión.
\end{abstract}

Palabras clave: Ergonomía, formación en ergonomía, desempeño profesional del ergónomo.

\begin{abstract}
This article considers the development of the profession in Argentina based on a national historical review of scientific publications, legislation and education offer. Then the totality of the 60 final works produced between 2014 and 2020 by students trained in ergonomics at the National Technological University - Regional Faculty of Buenos Aires was analyzed. The study was based on descriptive statistics as a method to identify its most important characteristics and the results highlighted the manufacturing industry as the main branch of activity in which they were developed. The people investigated in the origin of these works were the decision makers (supervisors, team leaders, managers and directors) and the expert members of these organizations (human resources, occupational medicine, quality, safety and hygiene). The objectives of the work carried out were oriented towards solving existing problems, most of them related to physical ergonomics to reduce accidents and musculoskeletal disorders at work. The article ends by opening up the reflection on the
\end{abstract}

1Universidad Tecnológica Nacional. Buenos Aires, Argentina. Correo electrónico: lnouviale@frba.utn.edu.ar. Orcid: https://orcid.org/oooo-0oo2-2761-0672 ${ }^{2}$ Universidad Tecnológica Nacional. Buenos Aires, Argentina. Correo electrónico: esthergiraudo1@gmail.com. Orcid: https://orcid.org/oooo-0o01-8807-7632 
strategies to improve the educational offer and the quality of the ergonomics career in order to accompany the development of the profession.

Keywords: Ergonomics, ergonomics education, professional performance of the ergonomist.

Recepción: 30.11.2020 / Revisión: 03.12.2020 / Aceptación: 09.12.2020

\section{Introducción}

El desarrollo de la ergonomía es un tema de reflexión para los miembros de su comunidad científica y profesional tanto en el nivel internacional como nacional. De hecho, la Asociación Internacional de Ergonomía ([IEA], s.f.) tiene como misión principal promover el desarrollo de la ergonomía en tanto disciplina o profesión. En la República Argentina, la Asociación de Ergonomía Argentina ([ADEA], s.f.) establece como primer propósito en su estatuto "propender al estudio, investigación, divulgación y efectiva aplicación de la ergonomía". Varios miembros expresan su preocupación por una visión acotada sobre el aporte del ergónomo y de la ergonomía (Dul et Al., 2012; Carpovich, 2020). A fin de favorecer su desarrollo, Dul y sus colaboradores presentan unas bases estratégicas para fortalecer e incrementar la calidad de la demanda y la aplicación de la ergonomía. Surge entonces la interrogante sobre el estado actual de su desarrollo en la Argentina y fue así como se analizan los antecedentes en términos de publicaciones, legislación y formación profesional.

En cuanto a la producción de documentos resultantes de trabajos e investigaciones, se citan algunos de los autores, universidades o editoriales que participaron a la difusión de la ergonomía en el país. Slemenson (2012) menciona indicios a partir del año 1964 con la publicación de un capítulo sobre la ergonomía en la revista de la Sociedad de Medicina del Deporte y el Trabajo. En la década de 1980, distintas universidades del país en ciudades como Rosario, La Plata, Tucumán, Mendoza, Buenos Aires, Córdoba, dieron lugar a la creación de laboratorios de ergonomía y ciencias afines que generaron cuadernos y otras publicaciones en gran parte desarrolladas en el campo de la ergonomía de producto (Hiba, 2020). A partir del 1984, el Centro de Estudios e Investigaciones Laborales - Programa de Investigaciones Económicas sobre Tecnología, Trabajo y Empleo (CEIL-PIETTE) acompaña este auge con la difusión de investigaciones y conocimientos sobre las condiciones de trabajo. Este centro comenzó a organizar seminarios y a editar publicaciones de ergonomía en lengua española con el recibimiento de Alain Wisner en 1987 (Slemenson, 2012). Durante este mismo periodo, Melo (2005) inició su carrera de docente universitario escribiendo textos que sirvieron de base para sus futuras publicaciones sobre el diseño de herramientas, la evaluación de puestos o el ambiente de trabajo, entre otros (Unión Latinoamericana de Ergonomía, s.f). Durante los años 2000, la Fundación Social Aplicada del Trabajo suma la publicación de libros a la literatura nacional sobre condiciones de trabajo y ergonomía. En 2019, la ADEA edita su primer libro: "Ergonomía Argentina: historia, miradas y aplicaciones", que hasta hoy es la obra nacional que recopila la mayor cantidad de trabajos, con la participación de 28 autores. En dieciséis capítulos dedicados a la aplicación de la ergonomía, diez de ellos mencionan seis ramas diferentes de actividad: las actividades primarias, los servicios sociales y salud, la industria manufacturera, la administración pública y defensa, la enseñanza y el transporte, almacenaje y comunicaciones. Los demás 
capítulos corresponden a temas teóricos así como metodológicos y no especifican una rama de actividad (ADEA, 2019).

En la legislación, la palabra ergonomía surge por primera vez en el decreto 1288/97 que describe el ejercicio de la kinesiología. Después de tres nuevas menciones en textos sobre seguridad, salud y riesgos del trabajo, una perspectiva del alcance de la disciplina se vislumbra en la resolución $\mathrm{n}^{0}$ 295/03 del Ministerio de Trabajo, Empleo y Seguridad Social, esta vez dentro del rubro de la higiene y seguridad en el trabajo como lo marca el título de dicha resolución. Se reconocen así los aportes de la ergonomía en la prevención de trastornos músculo-esqueléticos relacionados con el trabajo, y se hace una referencia a cuestiones organizacionales y psicosociales. Recién en 2015, el "Protocolo de ergonomía" (resolución $\mathrm{n}^{0} 886 / 15$ ) identifica centralmente los factores físicos. En 2018, la Superintendencia de Riesgos del Trabajo (SRT) realiza una encuesta sobre las condiciones de trabajo contemplando la organización del trabajo, los factores medioambientales, los riesgos psicosociales y los cambios tecnológicos. Difunde una sección denominada "factores disergonómicos", evidenciando que los trabajadores están expuestos a vibraciones (13,2\%), manipulación de cargas (20,3\%), movimientos repetitivos (49,9\%) y posturas forzadas (24,5\%). Si bien la ergonomía abarca un amplio espectro de trabajo, la legislación argentina asocia la ergonomía en varias oportunidades al campo físico.

La diversidad de capacitaciones en ergonomía ofrecidas en el ámbito universitario e institucional varía de manera significativa en cantidad de horas y contenidos. En cuanto a tipos de formación, aparece representada como: a) materia electiva u obligatoria en carreras de grado (ingeniería, diseño industrial, entre otros), b) cursos puntuales de duración inferior a 30 horas, c) cursos de entre 30 y 300 horas de duración, d) 1 (un) diplomado de más de 300 horas de duración (Universidad Nacional del Centro de la provincia de Buenos Aires), e) 1 (una) especialización en ergonomía de 400 horas de duración (Universidad Tecnológica Nacional - regional Buenos Aires). Los contenidos varían en función de esas cargas horarias y van desde pautas para aplicar la legislación vigente, los conocimientos para abordar la carga física, los conocimientos sobre los diferentes campos de aplicación, el diseño, los modelos teóricos, la metodología, los métodos y las técnicas, entre otros. Algunas formaciones profundizan diferentes campos de la ergonomía otras ponen el acento en el campo físico y la aplicación de métodos.

Vistos los antecedentes históricos, la gestión de acciones locales favorece el desarrollo continuo de la ergonomía desde hace casi seis décadas. Durante los últimos años, la orientación enfatiza el campo físico en el ámbito laboral, para resolver problemas que tienen efectos sobre la seguridad y la salud de los trabajadores, cuya necesidad de intervenciones respectivas es incuestionable. Sin embargo, esta investigación está motivada por la posibilidad para otros tipos de intervenciones, como por ejemplo: tratar las cuestiones organizacionales o de fiabilidad, trabajar en proyectos industriales o arquitectónicos. Precisamente el presente estudio tiende a obtener elementos para orientar las estrategias de desarrollo de la práctica profesional en ergonomía por intermedio de la formación profesional en la Argentina. Las posibilidades de promoción de la disciplina está orientada hacia la identificación de las ramas de actividad actuales y potenciales, la identificación de los tipos de intervención actuales y el conocimiento de los interlocutores de las organizaciones 
que originan estos trabajos de intervención. Por lo tanto, se definen asimismo los siguientes objetivos de este estudio:

\section{Objetivo general}

- Analizar los campos de aplicación de los trabajos finales integradores de alumnos de la carrera de la Universidad Tecnológica de la Facultad Regional Buenos Aires (UTN-FRBA) a fin de proponer estrategias de desarrollo para mejorar la formación en ergonomía.

\section{Objetivos específicos}

- Determinar las ramas de actividades abordadas por los alumnos en ergonomía a partir del análisis de 60 trabajos finales integradores presentados entre los años 2014 y 2020.

- Identificar el tipo de intervención según las demandas, los temas abordados y los interlocutores solicitantes de las organizaciones.

- Verificar la relación entre la formación recibida y los campos abordados en las intervenciones.

- Proponer estrategias de desarrollo de la profesión y de la disciplina desde la formación profesional en ergonomía de la UTN-FRBA.

\section{Conceptos relacionados con el estudio}

\section{La especialización en ergonomía de la Universidad Tecnológica de la Facultad Regional Buenos Aires}

La especialización en ergonomía es una carrera universitaria de la facultad regional Buenos Aires de la República Argentina (UTN). Comenzó a dictarse en el año 2007 (ordenanza $\mathrm{n}^{\mathrm{0}}$ 1105). Acreditada en dos oportunidades por la Comisión Nacional de Evaluación y Acreditación Universitaria (resolución CONEAU n ${ }^{0}$ 482/16), la especialización cumple con la ley de Educación Superior (ley $\mathrm{n}^{0}{ }^{24.521}$ ) que exige la acreditación de las carreras de posgrado. En 2017, el consejo superior aprueba la actualización del diseño curricular mediante ordenanza (ordenanza C.S. $\mathrm{n}^{\circ}$ 1580/17). La nueva estructura curricular cuenta con 15 seminarios organizados en 5 ejes: fundamentos, dimensión física, dimensión organizacional y cognitiva, dimensión metodológica y práctica profesional para la concepción de sistemas (UTN-FRBA, s.f.). El nuevo plan de estudios propone brindar herramientas teóricas y metodológicas para construir modalidades de intervención de los diferentes campos de la ergonomía: físico, organizacional y cognitivo. Asimismo, el mismo tiene una carga horaria de 400 horas de las cuales 360 horas se realizan de manera presencial, están dedicadas a los contenidos teóricos (165 horas) y a los trabajos prácticos con el acompañamiento docente (125 horas). Las 40 horas restantes están atribuidas al seminario "práctica profesional" que sirve de base para redactar el trabajo final integrador (TFI) (UTNFRBA, 2014). 


\section{El trabajo final integrador}

El TFI es de carácter obligatorio estipulado por las normativas de posgrado de la Facultad (Circular $\mathrm{N}^{\mathrm{o}}$ 3/14) y los alumnos deben aprobarlo para obtener el título de "Especialista en Ergonomía” con validez nacional (Resolución PEN 145/2018). La actividad de campo realizada en el marco del espacio curricular "práctica profesional" está articulada con los seminarios "taller de orientación de la práctica" y el "seminario integrador" que aseguran el acompañamiento de los alumnos durante varios encuentros áulicos. Los objetivos específicos de la práctica profesional se encuentran dentro de las líneas comunes de trabajo definidas por la carrera, acordadas entre la empresa, el alumno y el docente del seminario. Puede ser realizado en ámbitos propuestos por los estudiantes o bien en el marco de los distintos convenios con los que cuenta la institución. Su objeto es integrar en un tema, problema o proyecto específicos, los conceptos aprendidos durante el desarrollo de la carrera y vinculados a la experiencia profesional. Incluye la elaboración de un diagnóstico en base al análisis del problema o demanda institucional e incluso la presentación de propuestas de mejora.

\section{La demanda de intervención}

La intervención en ergonomía empieza y se construye a partir de la demanda. Las diferentes propuestas de esquemas de la metodología de intervención coinciden con la primera fase: el análisis de la demanda (Guérin et al., 2009; Vézina et al., 2016). Los autores del libro "Comprender el trabajo para transformarlo" consideran dos tipos de intervención en ergonomía: a) las demandas centradas en proyectos de concepción que transforman de manera significativa el trabajo; b) las demandas centradas en la resolución de problemas para tratar preguntas puntuales que suelen escalar en su nivel de importancia con el paso del tiempo. Si bien la población en el origen de la demanda puede ser doble: la dirección o los asalariados, las personas que formulan las demandas de intervención se pueden clasificar según las categorías de los interlocutores interesados definidas por Dul et al. (2012): a) operadores del sistema (operarios, delegados), b) expertos del sistema (ingenieros, recursos humanos, médicos, seguridad e higiene, etc.), c) decisores (supervisor, jefe, gerente, director), d) influyentes del sistema (aseguradoras de riesgos del trabajo, superintendencia de riesgos del trabajo, etc.). Estos interlocutores expresan intereses en términos de resultados para la empresa (disminuir el ausentismo, alcanzar la producción, etc.) o de efectos sobre la salud de las personas miembros del sistema (mejorar la salud física y mental, etc.).

\section{Las ramas de actividad}

La administración federal de ingresos públicos (AFIP) es un organismo de recaudación de fondos del estado argentino. Utiliza una clasificación especifica por rama de actividad a fin de regular los derechos y atribuciones de los contribuyentes (resolución AFIP $\mathrm{n}^{0}$ 3537). A continuación, se desglosa la lista de las ramas que se toman para el estudio con sus respectivas definiciones. 
Tabla 1. Clasificación de las ramas de actividad y su descripción.

\begin{tabular}{|c|c|c|}
\hline & $\begin{array}{l}\text { Nombre de la rama } \\
\text { de actividad }\end{array}$ & Descripción \\
\hline 1 & Actividades primarias & $\begin{array}{l}\text { Agricultura, ganadería, caza y silvicultura, pesca y servicios } \\
\text { conexos, explotación de minas y canteras. }\end{array}$ \\
\hline 2 & $\begin{array}{l}\text { Industria } \\
\text { manufacturera }\end{array}$ & $\begin{array}{l}\text { Industria realizando procesos de transformación y elaboración } \\
\text { de materias primas, sustancias orgánicas e inorgánicas en } \\
\text { productos, y de armado y terminación de productos. }\end{array}$ \\
\hline 3 & Construcción & $\begin{array}{l}\text { Actividad que abarca el movimiento de suelos, la preparación de } \\
\text { terrenos para obras, la demolición y voladura de edificios, } \\
\text { perforación y sondeo- excepto para la extracción de petróleo, gas } \\
\text { y la perforación de pozos hidráulicos. }\end{array}$ \\
\hline 4 & Comercio & $\begin{array}{l}\text { Actividad comercial mayorista, acopio, abastecimiento, } \\
\text { fraccionamiento, redistribución y actividad comercial al por } \\
\text { menor en la reventa al público de productos para consumo o uso } \\
\text { personal y doméstico. }\end{array}$ \\
\hline 5 & Hoteles y restaurantes & $\begin{array}{l}\text { Hospedaje temporal y servicios que comprende la venta de } \\
\text { comidas y bebidas preparadas para su consumo inmediato o } \\
\text { para llevar. Incluye la provisión de comida preparada para } \\
\text { empresas. }\end{array}$ \\
\hline 6 & $\begin{array}{l}\text { Transporte, } \\
\text { almacenaje } \\
\text { comunicaciones }\end{array}$ & $\begin{array}{l}\text { Actividades de transporte de cargas y de pasajeros por vías } \\
\text { terrestre, acuática y aérea, así como las actividades de transporte } \\
\text { auxiliares. }\end{array}$ \\
\hline 7 & $\begin{array}{l}\text { Servicios financieros, } \\
\text { inmobiliarios, } \\
\text { alquileres } \\
\text { empresariales }\end{array}$ & $\begin{array}{l}\text { Actividades de intermediación monetaria y financiera. } \\
\text { Comprende las actividades de servicios de seguros de salud, } \\
\text { seguros de vida, generales, servicios de aseguradoras de riesgo } \\
\text { de trabajo, etc. Se incluyen las operaciones con inmuebles } \\
\text { propios y de terceros. }\end{array}$ \\
\hline 8 & $\begin{array}{l}\text { Administración } \\
\text { pública y defensa }\end{array}$ & $\begin{array}{l}\text { Servicios generales de la administración pública, prestación } \\
\text { pública de servicios a la comunidad en general (de asuntos } \\
\text { exteriores, defensa, de orden público y seguridad y de protección } \\
\text { civil) y los servicios de la seguridad social obligatoria. }\end{array}$ \\
\hline 9 & Enseñanza & $\begin{array}{l}\text { Enseñanza oficial y privada de todo tipo impartida por } \\
\text { instituciones y por profesores particulares, como la enseñanza de } \\
\text { tiempo completo, de tiempo parcial y de carácter intensivo, en } \\
\text { horario diurno y nocturno, de cualquier nivel y para cualquier } \\
\text { profesión. }\end{array}$ \\
\hline 10 & $\begin{array}{l}\text { Servicios sociales y de } \\
\text { salud }\end{array}$ & $\begin{array}{l}\text { Actividades relacionadas con la salud humana como los servicios } \\
\text { hospitalarios, de atención ambulatoria, atención domiciliaria } \\
\text { programada, servicios odontológicos, de diagnóstico y de } \\
\text { emergencias y traslados. }\end{array}$ \\
\hline 11 & Trabajo doméstico & $\begin{array}{l}\text { Actividades de hogares privados que emplean personal } \\
\text { doméstico de todo tipo como cocineros, mayordomos, choferes, } \\
\text { lavanderas, niñeras, etc. }\end{array}$ \\
\hline 12 & $\begin{array}{l}\text { Servicios } \\
\text { comunitarios, sociales } \\
\text { y personales }\end{array}$ & $\begin{array}{l}\text { Servicios de recolección y tratamiento de basura, barrido y } \\
\text { limpieza de calles, caminos, etc. Actividades de organizaciones } \\
\text { empresariales, profesionales, de sindicatos, empleadores y las } \\
\text { organizaciones religiosas y políticas. Servicios de diversión, } \\
\text { esparcimiento y culturales - teatro, espectáculos, etc. Servicios } \\
\text { de lavandería y servicios personales directos -belleza, estética } \\
\text { corporal, etc. }\end{array}$ \\
\hline 13 & Otras ramas & Electricidad, gas y agua. \\
\hline
\end{tabular}

Fuente: adaptado del AFIP (2013). 


\section{Materiales y métodos}

La metodología utilizada en este estudio comprende análisis estadísticos descriptivos, también llamados exploratorios, los cuales reúnen los datos que permiten detectar un patrón de comportamiento general, así como partes del mismo, y darlos a conocer mediante gráficos y su interpretación.

Las técnicas de la estadística descriptiva permiten reducir la masa de datos a unos pocos indicadores con los que se puede describir adecuadamente el comportamiento de las variables.

En función del tamaño de la población objeto de estudio, se pueden obtener datos completos de sus elementos lo cual permite el análisis estadístico descriptivo.

Para concretar este estudio se cumplieron los siguientes pasos:

1- Selección de la población a estudiar en su totalidad, o sea los 60 TFI presentados por los estudiantes de ergonomía desde el año 2014 al 2020.

2- Establecimiento de las características a analizar de la población sobre tres aspectos: identificar las ramas de actividad, el tipo de intervención y los interlocutores. Para agrupar los datos obtenidos según esas clasificaciones y establecer un patrón común se recurre a fuentes reconocidas oficialmente (AFIP, SRT, etc.).

3- Toma de datos de cada TFI según las características determinadas mencionadas en el punto 2.

4- Análisis de datos y presentación mediante gráficos.

5- Exposición de los resultados y conclusiones.

\section{Resultados}

En primer lugar se identificaron las ramas de actividad elegidas por los alumnos en formación en ergonomía para investigar, y por defecto las ramas no desarrolladas. Luego se identificó el tipo de intervención según la demanda y por último los interlocutores de las organizaciones que expresaron las demandas. A continuación se detallan los resultados obtenidos:

1. La industria manufacturera: Primera rama de actividad en el origen de las intervenciones en ergonomía

La clasificación de los trabajos finales según las ramas de actividad (resolución AFIP $\mathrm{n}^{0} 3537$ ) permite comparar la repartición de la población de trabajadores de la Argentina y la distribución de los trabajos presentados. 
Tabla 2. Clasificación de los TFI por ramas de actividad.

\begin{tabular}{|c|c|c|c|c|c|}
\hline & $\begin{array}{l}\text { Porcentaje de } \\
\text { trabajadores }\end{array}$ & $\begin{array}{l}\text { Resolución } \\
\text { de problema }\end{array}$ & Proyecto & Total & Porcentaje \\
\hline $\begin{array}{l}\text { Actividades } \\
\text { primarias }\end{array}$ & 3,3 & 1 & 1 & 2 & 3,3 \\
\hline $\begin{array}{l}\text { Industria } \\
\text { manufacturera }\end{array}$ & 10,0 & 31 & 2 & 33 & 55,0 \\
\hline Construcción & 9,5 & $\mathrm{O}$ & $\mathrm{O}$ & 0 & 0,0 \\
\hline Comercio & 17,9 & 0 & $\mathrm{O}$ & $\mathrm{O}$ & 0,0 \\
\hline $\begin{array}{l}\text { Hoteles y } \\
\text { restaurantes }\end{array}$ & 4,5 & $\mathrm{O}$ & o & $\mathrm{O}$ & 0,0 \\
\hline $\begin{array}{l}\text { Transporte, } \\
\text { almacenaje y } \\
\text { comunicaciones }\end{array}$ & 6,8 & 9 & 1 & 10 & 16,7 \\
\hline $\begin{array}{l}\text { Servicios financieros, } \\
\text { inmobiliarios, } \\
\text { alquileres y } \\
\text { empresariales }\end{array}$ & 9,9 & 3 & $\mathrm{O}$ & 3 & 5,0 \\
\hline Enseñanza & 9,7 & 1 & $\mathrm{O}$ & 1 & 1,7 \\
\hline $\begin{array}{l}\text { Servicios sociales y } \\
\text { de salud }\end{array}$ & 6,5 & 6 & $\mathrm{O}$ & 6 & 10,0 \\
\hline Trabajo doméstico & 8,1 & 0 & 0 & 0 & 0,0 \\
\hline $\begin{array}{l}\text { Otros servicios } \\
\text { comunitarios, } \\
\text { sociales y personales }^{1}\end{array}$ & 5,5 & 1 & o & 1 & 1,7 \\
\hline $\begin{array}{l}\text { Administración } \\
\text { pública y defensa }\end{array}$ & 7,6 & $\mathrm{O}$ & $\mathrm{O}$ & $\mathrm{O}$ & 0,0 \\
\hline Otras ramas & 0,7 & 2 & 2 & 4 & 6,7 \\
\hline Total & 100,0 & 54 & 6 & 60 & 100,0 \\
\hline
\end{tabular}

Fuente: elaboración propia.

La industria manufactura está en el origen del 55\% de las intervenciones en ergonomía realizadas por los alumnos de la especialización en ergonomía.

Las ramas de actividad tales como la construcción, el comercio, los hoteles y restaurantes, la administración pública y defensa, no dieron origen a la intervención. Sin embargo, en 2018, la suma de la población ocupada en estas ramas de actividades representa el 39,5\% de los trabajadores (SRT, 2018). Estos resultados permiten plantear la razón de esta falta de demanda en ciertas ramas de actividad. Posiblemente se deba al desconocimiento, a la falta de difusión sobre la actividad del ergónomo, o la falta de cumplimiento de la legislación.

En este caso, los alumnos en formación trabajan en estas ramas de actividades o tienen un contacto familiar o cercano con el origen de la demanda de intervención para presentar el TFI. 
2. Demandas de intervención centradas en la resolución de problemas puntuales

Tal como lo deja entrever el temario del manual de ergonomía (Falzon, 2009), los campos de acción del ergónomo son diversificados. Puede trabajar en la concepción de sistemas y dispositivos de trabajo, en proyectos arquitectónicos, diseño de programas informáticos y en la prevención de riesgos profesionales entre otros. Sin embargo, la cantidad de trabajos realizados en proyectos de concepción es de 10\%, en la población estudiada. La clasificación de los trabajos finales según el tipo de demanda permite identificar los temas solicitados en la Argentina.

Tabla 3. Clasificación de los TFI por tipo de demanda.

\begin{tabular}{|l|l|c|c|}
\hline Tipo de demanda & Tema & Cantidad & Porcentaje \\
\hline \multirow{3}{*}{$\begin{array}{l}\text { Resolución de } \\
\text { problemas }\end{array}$} & Trastornos músculo esqueléticos & 30 & 50,0 \\
\cline { 2 - 4 } & Riesgos psicosociales en el trabajo & 3 & 5,0 \\
\cline { 2 - 4 } & Accidentes & 7 & 11,7 \\
\cline { 2 - 4 } & Discapacidad & $\mathbf{1}$ & 1,7 \\
\cline { 2 - 4 } & Errores & $\mathbf{2}$ & 3,3 \\
\cline { 2 - 4 } & Organización del trabajo & 9 & 15,0 \\
\cline { 2 - 4 } & Otros & $\mathbf{2}$ & 3,3 \\
\hline \multirow{3}{*}{ Proyecto } & Diseño de mobiliario & $\mathbf{2}$ & 3,3 \\
\cline { 2 - 4 } & Diseño de proceso industrial & $\mathbf{2}$ & 3,3 \\
\cline { 2 - 4 } & Modificaciones arquitectónicas & 2 & 3,3 \\
\hline Total & & 60 & 100,0 \\
\hline
\end{tabular}

Fuente: elaboración propia.

Los trabajos solicitados para resolver problemas existentes en las organizaciones tienden en su mayoría a la disminución de los accidentes $(11,7 \%)$ o los trastornos músculo esqueléticos (50\%) para evitar el ausentismo. Los demás temas tratan de los riesgos psicosociales en el trabajo, la discapacidad, los errores y la organización del trabajo. A continuación se citan algunos ejemplos transcritos en los TFI, expresados por las personas que solicitan las intervenciones:

- Resultados para la empresa: "reducir [...] el ausentismo que poseemos actualmente de los trabajadores", "produce inconvenientes organizacionales por los ausentismos generados, perjudicando el servicio brindado “, reducción del 9,6 \% de alícuota con la ART por accidentes de [...], etc.

- Efectos para las personas: “ disminuir la siniestrabilidad relacionada con la actividad de los operadores del sector [...]", "casos de tendinitis, síndrome de túnel carpiano y denuncias de trabajadores por dolores musculo esqueléticos, a pesar mejoras de tecnología del sector productivo", "la solicitud de realizar una intervención ergonómica surge del departamento médico como consecuencia de las reiteradas consultas de los trabajadores sobre molestias localizadas principalmente a nivel de miembros superiores", etc. 
- Adecuar las situaciones de trabajo: "adecuar espacios de trabajo", "revisar la manipulación de accesorios para el proyecto nuevo", "rediseño de los puestos con arquitectos teniendo en cuenta la actividad de los trabajadores., etc.

Las motivaciones que originaron las demandas están expresadas en términos de resultados para la empresa, efectos para las personas y adecuación de los componentes de la situación de trabajo.

\section{Decisores y expertos, los principales solicitantes de intervención en ergonomía}

El análisis de los interesados informa acerca de quiénes son los interlocutores solicitantes de una intervención ergonómica.

Tabla 4. Distribución de los trabajos finales por interlocutor al origen de la solicitud de intervención.

\begin{tabular}{|c|c|c|c|c|c|c|}
\hline & & $\begin{array}{l}\text { Resolución } \\
\text { de problema }\end{array}$ & Proyecto & $\begin{array}{c}\text { Cantidad } \\
\text { total }\end{array}$ & $\begin{array}{c}\text { Porcentaje } \\
\text { total }\end{array}$ & Porcentaje \\
\hline $\begin{array}{l}\text { Operadores } \\
\text { del sistema }\end{array}$ & $\begin{array}{l}\text { Operarios, } \\
\text { delegados, } \\
\text { sindicatos }\end{array}$ & 3 & 0 & 3 & 5,00 & 5,00 \\
\hline \multirow[t]{4}{*}{ Decisores } & Supervisor/a & 1 & $\mathrm{O}$ & 1 & \multirow{4}{*}{43,33} & 1,67 \\
\hline & Jefe/a & 7 & 1 & 8 & & 13,33 \\
\hline & Gerente & 6 & 1 & 7 & & 11,67 \\
\hline & Director/a & 7 & 3 & 10 & & 16,67 \\
\hline \multirow[t]{4}{*}{ Expertos } & Calidad & 1 & $\mathrm{O}$ & 1 & \multirow{4}{*}{48,33} & 1,67 \\
\hline & $\begin{array}{l}\text { Medicina } \\
\text { laboral }\end{array}$ & 5 & 0 & 5 & & 8,33 \\
\hline & $\begin{array}{l}\text { Seguridad e } \\
\text { higiene }\end{array}$ & 15 & 1 & 16 & & 26,67 \\
\hline & RRHH & 7 & $\mathrm{O}$ & 7 & & 11,67 \\
\hline Influyentes & $\begin{array}{l}\text { ART, SRT, } \\
\text { etc. }\end{array}$ & 1 & 0 & 1 & 1,67 & 1,67 \\
\hline $\begin{array}{l}\text { No } \\
\text { especifica }\end{array}$ & & 1 & 0 & 1 & 1,67 & 1,67 \\
\hline Total & & 54 & 6 & 60 & 100,00 & 100,00 \\
\hline
\end{tabular}

Fuente: elaboración propia.

Si se analiza el cuadro, se observa que los operarios tienen poca participación en la formulación de las demandas (5\%) a pesar de ser los protagonistas de los problemas a resolver. En cambio los que recurrieron a la demanda fueron: uno, el cuerpo de delegados del sector; otro, los operadores de un sector, ambos por problemas de orden físico. En otro de los casos, el sindicato solicita intervención para resolver problemas de riesgos psicosociales.

El 43,33 \% de los trabajos tienen como comitente a los tomadores de decisión (supervisores, jefes, gerentes, directores), quienes son los solicitantes de proyectos (5 sobre 6 de la población investigada). 
El 48,33\% provienen de los expertos de las organizaciones (médico, responsable de seguridad e higiene, de recursos humanos, personal del sector de calidad). De los 28 solicitantes, 16 personas son responsables de la Seguridad e higiene.

Los influenciadores solicitan resolver un problema de salud para disminuir el ausentismo por el costo que ello implica, por ser en un caso miembro de la aseguradora de riesgos del trabajo.

\section{Conclusiones}

Se considera que el amplio campo de acción de la ergonomía puede beneficiar a sus profesionales ya que abre la posibilidad de tener como objeto de intervención el diseño de productos, de programas informáticos y de procesos de bienes o servicios. Sin embargo, el presente estudio de los 60 TFI demostró que todos los trabajos se realizaron en el ámbito laboral para diseñar o mejorar los procesos de bienes o servicios. Posiblemente, se puede atribuir esto a la estructura curricular de la especialización, dado que solo en el seminario "ergonomía de concepción de sistemas de trabajo y producto" se aborda el diseño de producto. Las carreras de base de los estudiantes (seguridad e higiene, ingeniería, kinesiología, terapia ocupacional, psicología, sociología) tampoco están orientadas hacia ese campo de intervención.

Con respeto a las ramas de actividad analizadas en este estudio, la industria manufacturera está en el origen de la mitad de las intervenciones en ergonomía. No obstante, las ramas que aún no solicitan intervenciones como la construcción, el comercio, los hoteles y restaurantes, la administración pública y defensa, podrían beneficiarse con el aporte de ergónomos, por ejemplo en proyectos de edificación o de transformación. En este sentido, identificamos en el estudio que los decisores de mayor nivel jerárquico (directores o gerentes) son los interlocutores interesados en integrar a los ergónomos en los proyectos de concepción.

Si bien el campo físico está más requerido en este estudio para evitar enfermedades del trabajo y accidentes, estos problemas tienen repercusiones en ciertos cambios organizacionales. Más allá de que se busque mejorar la salud y la seguridad en el trabajo, el objetivo final de los interlocutores apunta a evitar ausencias y lograr mayor productividad. Si se miran las intervenciones sobre la disminución de "errores", la "carga mental" o la "fiabilidad" de las instalaciones, la cantidad es escasa, aunque el seminario "ergonomía cognitiva" existe desde los inicios de la formación. Con el cambio curricular realizado en 2017, el cual agregó un seminario sobre los factores psicosociales en el trabajo, los estudiantes comenzaron a realizar intervenciones para abordar los problemas de esa índole.

A la luz de estos resultados, se considera oportuno reflexionar sobre la formación, difusión y comunicación de los diferentes campos de la ergonomía, su aplicabilidad en las distintas ramas de actividad y la prevención desde las etapas de diseño.

Se plantea si se debe actuar solamente desde la formación en ergonomía de la UTNFRBA, modificando la carga horaria de la carrera, ajustando los contenidos en términos de conocimientos y de metodología o si también se debe revisar el abanico de propuestas de 
formación en ergonomía en el nivel nacional para cubrir diferentes oportunidades de intervención. Para ello sería conveniente revisar las propuestas curriculares en los diferentes niveles de formación e incluir en las materias afines esos temas en la escuela primaria, secundaria, carreras de grado y no solamente en la oferta educativa de posgrado. También, se considera oportuno buscar la manera de dar mayor visibilidad a los aportes de los ergónomos y así lograr que los interlocutores comprendan la importancia de las intervenciones ergonómicas, especialmente en las ramas de actividad que aún no la solicitan. Otro eje de difusión a tener en cuenta es motivar a las organizaciones o instituciones que agrupan a los trabajadores según su oficio, como por ejemplo los sindicatos, las cámaras, etc.

En cuanto a la carrera de la UTN-FRBA, se observa que no alcanza integrar los contenidos teóricos para tener mayor cantidad de intervenciones en estos rubros (proyecto, error, factores psicosociales, etc.). En este caso, se debería revisar si los alumnos logran identificar posibles intervenciones con los conocimientos propuestos durante las clases o si se deben ajustar los contenidos teórico-prácticos.

La visibilidad que dio este estudio a los tipos de trabajo realizados, dispara también la reflexión sobre la necesidad de ampliar el abordaje de la ergonomía de concepción a fin de incentivar proyectos. Para ello es necesario profundizar la etapa de transformación, implementación de soluciones y seguimiento de proyectos que abarquen la interacción entre los distintos campos de la ergonomía ya que no son independientes. A fin de diversificar los tipos de trabajos integradores finales, se piensa en la incorporación de relatos sobre la práctica, dirigidos hacia los alumnos durante el transcurso de la formación, para motivarlos a elegir un tipo menos recurrente de intervención (adecuación de puestos de trabajo a personas con discapacidad, de los componentes de los sistemas de trabajo para evitar errores, etc.). Además, el aporte de conocimientos propios a la gestión de proyectos y las características técnicas inherentes a las materias de ingeniería contribuirían a una mejor integración de la disciplina en su casa de estudio, la Universidad Tecnológica Nacional.

Finalmente, estos resultados llevan a ratificar la preocupación por la "visión acotada de la ergonomía" y a proponer una reflexión colectiva sobre las estrategias para disminuir la brecha entre lo ideal y la realidad.

\section{Referencias}

Administración Federal de Ingresos Públicos (01 de noviembre 2013). Resolución General $\mathrm{n}^{\mathrm{o}} 3537 / 2013$. Nomenclador de actividades. Buenos Aires. http://biblioteca.afip.gob.ar/dcp/REAG01003537_2013_10_30

Asociación de Ergonomía Argentina (2019). Ergonomía Argentina: historia, miradas y aplicaciones. ADEA.

Asociación de Ergonomía Argentina (s.f.) Estatuto. Recuperado en fecha 4 de septiembre 2020 de: http://adeargentina.org.ar/estatuto/

Asociación Internacional de Ergonomía (s.f.) Mission \& Goals. Recuperado en fecha 4 de septiembre $2020 \mathrm{de}$ https://iea.cc/about/introduction/ 
Carpovich, M.L. (18 de agosto 2020). Consultoría y Asesoramiento en ergonomía , proceso de intervención en empresas. [Sesión de conferencia] Jornadas de actualización de ergonomía, Asociación de Ergonomía Argentina, Buenos Aires, Argentina.

Comisión Nacional de Evaluación y Acreditación Universitaria (1 de julio 2016). Resolución $n^{0} 482 / 2016$. Carrera $n^{0} 21.359 / 15$. https://www.coneau.gob.ar/archivos/resoluciones/Res482-16C21359-15.pdf

Congreso de la Nación Argentina (20 de julio 1995) Ley de educación superior $\mathrm{n}^{0} \mathbf{2 4 . 5 2 1}$. Disposiciones preliminares. Educación Superior. Educación superior no universitaria. Educación superior universitaria. Disposiciones complementarias y transitorias. Buenos Aires.

Dul, J., Bruder, R., Buckle, P., Carayon, P., Falzon, P., Marras, W.S., Wilson, J.R. \& van der Doelen, B. (2012). A strategy for human factors/ergonomics: Developing the discipline and profession. Ergonomics, 55(4), 377-395. https://doi.org/10.1080/00140139.2012.661087

Falzon, P. (2009). Manual de Ergonomía. Editorial Modus Laborandi.

Guérin, F., Laville, A., Daniellou, F., Duraffourg, J. \& Kerguelen A. (2009). Comprender el Trabajo para transformarlo. Práctica de la Ergonomía. Editorial Modus Laborandi.

Hiba, J.C., Ruibal, A., \& Belletini, O. (1 de septiembre 2020). Historia de la Ergonomia Argentina. En G. Cuenca (Moderador), Jornadas de actualización de ergonomía. Asociación de Ergonomía Argentina, Buenos Aires, Argentina.

Melo, J. L. (2005). Ergonomía. Ediciones Journal.

Ministerio de Trabajo, Empleo y Seguridad Social (21 de noviembre 2003). Resolución

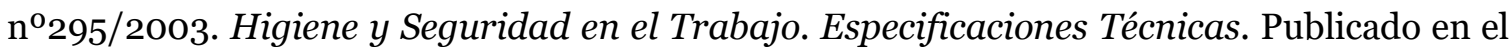
Boletín Oficial, Buenos Aires. https://www.argentina.gob.ar/normativa/nacional/resoluci\%C3\%B3n-295-2003-90396

Poder Ejecutivo Nacional (2 de diciembre 1997). Decreto nº 1288/97, Ejercicio de la Kinesiología. Publicado en el Boletín Oficial, Buenos Aires. http://servicios.infoleg.gob.ar/infolegInternet/anexos/45000-49999/47603/norma.htm

Poder Ejecutivo Nacional (12 de septiembre 2018). Resolución $\mathrm{n}^{0} 145 / 2018$. Validez Nacional título Universidad Tecnológica Nacional. https://www.frba.utn.edu.ar/wpcontent/uploads/2019/02/res145-18-Ergo-Validez-Nac-1.pdf

Slemenson, C. (2012). La ergonomía y el ergónomo: Un aporte para la construcción de la profesión en la República Argentina [tesis de maestría]. Repositorio Digital Institucional de la Facultad de Ciencias Sociales de la Universidad de Buenos Aires. http://www.ceilconicet.gov.ar/wp-content/uploads/2016/11/2013slemenson.pdf

Superintendencia de Riesgos del Trabajo (24 de abril 2015). Resolución nº886/2015. Protocolo de Ergonomía. Publicada en el Boletín Oficial, Buenos Aires. http://servicios.infoleg.gob.ar/infolegInternet/anexos/245000-249999/246272/norma.htm

Superintendencia de Riesgos del Trabajo (2018). Encuesta Nacional a trabajadores sobre Condiciones de Empleo, Trabajo, Salud y Seguridad (ECETSS) 2018. 
https://www.srt.gob.ar/observatoriosrt/wpcontent/uploads/2019/12/ECETSS Informe de Resultados.pdf

Unión Latinoamericana de Ergonomía (s.f.). Material ULAERGO. Prof. José Luis Melo Argentina $A D E A$. Consultado el 25 de noviembre 2020. http://www.ulaergo.com/Luis Melo.php

Universidad Tecnológica Nacional - Facultad Regional de Buenos Aires (s.f.). Estructura curricular ergonomía. Consultado el 4 de septiembre 2020. https://www.frba.utn.edu.ar/posgrado/estructura-curricular-ergonomia/

Universidad Tecnológica Nacional (17 de agosto 2006). Ordenanza del Consejo Superior $\mathrm{n}^{\mathrm{0}} 1105$. Aprueba la carrera de especialización en ergonomía en el ámbito de la Universidad tecnológica Nacional. http://csu.rec.utn.edu.ar/CSU/ORD/1105.pdf

Universidad Tecnológica Nacional - Facultad Regional de Buenos Aires. (23 de junio 2014) Circular 3/2014. Comunicar los alcances de la obligatoriedad del Trabajo Final de Especialización como requisito parar las carreras de Especialización de la FRBA. https://www.frba.utn.edu.ar/wp-content/uploads/2019/02/Circular-3-tfi-2014.pdf

Universidad Tecnológica Nacional (27 de abril 2017). Ordenanza del Consejo Superior $\mathrm{n}^{\mathrm{0}} 1580 / 17$. Actualización de la especialización en ergonomía en el ámbito de la Universidad tecnológica Nacional. http://csu.rec.utn.edu.ar/CSU/ORD/1580.pdf

Vézina, N., Chatigny, C., \& Calvet, B. (2016). L’intervention ergonomique: Que fait-on des caractéristiques personnelles comme le sexe et le genre ? Perspectives interdisciplinaires sur le travail et la santé, 18-2. https://doi.org/10.4000/pistes.4847 